\title{
Can accelerated expansion of the universe be due to spacetime vorticity?
}

\author{
Babur M. Mirza* \\ Department of Mathematics, \\ Quaid-i-Azam University, Islamabad 45320, Pakistan
}

November 15, 2018

\begin{abstract}
We present here a general relativistic mechanism for accelerated cosmic expansion and the Hubble's constant. It is shown that spacetime vorticity coupled to the magnetic field density in galaxies causes the galaxies to recede from one another at a rate equal to the Hubble's constant. We therefore predict an oscillatory universe, with zero curvature, without assuming violation of Newtonian gravity at large distances or invoking dark energy/dark matter hypotheses. The value of the Hubble's constant, along with the scale of expansion, as well as the high isotropy of CMB radiation are deduced from the model.
\end{abstract}

\section{Introduction}

Accelerated expansion of the universe, as observed, for example, in the cosmological redshift measurements using type-Ia supernovae (SNe Ia) as standard candles [1, 2], implies the need for an expansion energy effective at least up to the $M p c$ scale. A number of independent observations (including the SNe Ia redshift, the Hubble's constant measurements [3-5], the cosmic microwave background (CMB) [6,7], baryon acoustic oscillations [8,9], and various cosmological probes [10]), have measured the contributions of matter and the cosmological constant to the energy density of the universe, providing an accurate measurement of the cosmic acceleration [11]. However the amount of energy for this acceleration implies a hidden or dark form of energy which is approximately three times of the observed gravitational mass-energy density in the universe.

Within Einstein's general theory of relativity, the observed expansion rate can be accounted for by including a cosmological constant, whose origin remains somewhat mysterious. In this context various mechanisms have been postulated, including new forms of hypothetical particles, or modifications of the NewtonianEinsteinian law of gravitation at large distances, among others. However these

*E-mail: bmmirza2002@yahoo.com 
theories are specialized in the sense that they fail to account for other observed features of the universe, such as the high degree of isotropy in CMB, or even some feature of the expansion, such as the correct value of the Hubble's constant.

In this work we show that the specific form of the cosmological constant, hence cosmic acceleration, can be described by spacetime vorticity, generated by galactic rotations. We show that this vorticity coupled to the local (galactic) magnetic field provides the requisite push (repulsive energy) causing the individual galaxies to recede at an accelerated rate. We are therefore led to an oscillatory universe, where expansion and conversely contraction rate is determined by local spacetime vorticity, rather than global geometry (curvature) of the spacetime. Although local perturbation effects render it difficult to determine nature of the large scale curvature of the universe, present astronomical observations constrain the spatial curvature of the universe to be very close to zero [12-16] with less than $0.4 \%$ experimental error. This implies that where as the local spacetime geometries around galaxies must be determined from the general theory of relativity, the large scale structure is well described by Friedmann equations with zero spatial curvature, or equivalently by Newtonian cosmology in three dimensional Euclidean space. In this spacetime vorticity, rather than curvature, has the dominant cumulative effect on the accelerated expansion. We show that locally generated galactic magnetic fields provide the essential feedback in the cosmic acceleration, which collectively extends to the $M p c$ scale.

Large scale magnetic fields have been detected in the intergalactic medium surrounding galactic clusters [17-21]. These intracluster magnetic fields can be generated by turbulent gas motions due to massive interactions between galaxies and the intracluster gas, by galactic winds, and by jets from active galactic nuclei . Although generated locally within galaxies, these fields extend much farther in space. Microgauss magnetic fields have been observed in the intracluster medium of a number of rich clusters, where the estimates of the regular magnetic field strength for clusters range from $0.2-3 \mu G$ [22-25] and on supercluster scales, such as for the Coma cluster and the Abell 1367 cluster (distance scale $40 M p c$ ) [26] of the order $0.2-0.6 \mu G$. Observations of magnetic fields in the Coma supercluster and in redshift $z \simeq 2$ radio galaxies also hint at the existence of widespread cosmological fields [21]. Magnetic field energy density per galaxy is therefore generally quite large, comparable to the total matter density in each galaxy. Also these microgauss fields extend up to many $M p c$ without any substantial decaying. This requires a general amplification mechanism, effective up to very large cosmic distances.

The above observations motivate a magneto-vorticity interpretation for the energy responsible for the cosmological constant. We shall see below that this coupling of magnetic energy density and spacetime vorticity gives the precise form of the expansion as actually observed. At the same time the coupling of the locally generated magnetic fields and the spacetime vorticity due to galactic rotation causes the locally prevalent (intraclusteral) magnetic field energy to extend up to the Gpc scales without decaying. We show that this leads to the correct (observed) value of the Hubble's expansion rate, the scale of cosmic 
expansion, and the requisite amount of missing energy (three times the observed matter density), without invoking the dark energy/dark matter hypotheses, or modifying Newtonian-Einsteinian law of gravity at large distances. We also show that the discrepancy in the observed value of the Hubble's constant is due to the inclusion or exclusion of magneto-vorticity effects in the observational scales involved.

\section{The cosmological model}

Galactic systems, such as galactic clusters, have a residual magnetic field generated in individual galaxies with non-zero angular momentum. It can be shown, using general relativistic Maxwell equations in axially symmetric spacetimes (see [27], and references therein), that for a mass distribution $M$, rotating with an angular momentum $J$, the magnetic field coupled to spacetime vorticity has the form

$$
B=-\frac{B_{0}}{r \sqrt{1-\frac{R_{s}}{r}}} \cos (\varphi-\omega t), r>R_{s} .
$$

where $R_{s}=2 G M / c^{2}$. Using the total energy density equation $\epsilon_{B}=\int_{0}^{R} 4 \pi r^{2} B^{2} d r$, up to a radius $R$, we find after integrating $\xi=\varphi-\omega t$, from 0 to $\pi$, that the total energy density due to the magneto-vorticity field (1) is given by,

$$
\epsilon_{B}=4 \pi B_{0}^{2}\left[R+R_{s} \ln \left(R-R_{s}\right)\right], R>R_{s} .
$$

With the magnetic energy density $\epsilon_{B}$ along the radial direction $R$ per unit mass, and total energy constant $-k / 2$ per unit mass, we therefore have by energy conservation,

$$
v^{2}-\frac{2 G M}{R}+\frac{E_{B}}{4 \pi}=-k
$$

where $v$ is the expansion velocity, related to the Hubble expansion rate $H$ by $v=H R$, with $R$ being the scale factor. This gives the equation

$$
H^{2}-\frac{8 \pi G}{3} \rho+\epsilon_{B}=-\frac{k}{a^{2}},
$$

where $H(t)=\dot{R}(t) / R(t)$, and $\epsilon_{B}=E_{B} / 4 \pi R^{2}$ is the magnetic energy density, where we have taken $c=1$. Also, for a flat universe, we take $k=0$. Here $\rho$ is the ordinary matter density.

Acceleration equation for the expansion rate can be derived by differentiating the energy equation (3). This has two contributions to the total acceleration. First, velocity due to the gravitational potential energy is given by,

$$
v_{G}^{2}=-\frac{8 \pi}{3} G \rho(t) R(t)^{2} .
$$

Using mass conservation equation $\rho_{0} R_{0}^{3}=\rho R^{3}$ we thus have for the gravitational acceleration, by differentiating (5),

$$
a_{G}=-\frac{4 \pi}{3} G \rho R<0 .
$$


Also, the other contribution to acceleration comes from the magnetic energy density. We see that this energy corresponds to the kinetic energy per unit mass given by $\epsilon_{B}=v_{B}^{2} / 2$, therefore,

$$
v_{B}^{2}=4 \pi B_{0}^{2}\left[R+R_{s} \ln \left(R-R_{s}\right)\right], R>R_{s} .
$$

Since $R>>R_{s}$, we write $\ln \left(R-R_{s}\right) \approx \ln R$. Also since acceleration $a_{B}=$ $d v_{B} / d t=(1 / 2) d v_{B}^{2} / d R$, we have for the acceleration corresponding to this velocity

$$
a_{B}=\frac{d^{2} R}{d t^{2}}=4 \pi B_{0}^{2}\left[1+\frac{2 G M}{c^{2} R}\right], R>0 .
$$

Putting for total mass in the disk, $M=2 \pi \rho R^{2}$, and using equipartition of energy $B_{0}^{2} / 3$ in the three dimensional space, we get

$$
a_{B}=4 \pi B_{0}^{2}+\frac{16 \pi^{2} B_{0}^{2} G}{3 c^{2}} \rho R, R>0 .
$$

The total acceleration $\left(a_{G}+a_{B},\right)$ in any direction, is therefore,

$$
\frac{d^{2} R}{d t^{2}}=-\frac{8 \pi}{3} G \rho\left[1-\frac{2 \pi B_{0}^{2}}{c^{2}}\right] R+4 \pi B_{0}^{2}, R>0 .
$$

The acceleration (10) equation has real solution for uniform constant mass density, given by

$$
R(t)=A \cos \alpha t+\frac{4 \pi B_{0}^{2}}{\alpha^{2}},
$$

where $\alpha=\sqrt{8 \pi G \rho\left(1-2 \pi B_{0}^{2} / c^{2}\right) / 3}$. Here $\alpha$ can be real or imaginary corresponding to $\left(1-2 \pi B_{0}^{2} / c^{2}\right)$ is negative or positive. Putting $R(0)=0$, we have $A=-4 \pi B_{0}^{2} / \alpha^{2}$, therefore we have the branches for the solution

$$
\begin{aligned}
R(t) & =\frac{4 \pi B_{0}^{2}}{\beta^{2}}(1-\cosh \beta t), 4 \pi B_{0}^{2}>c^{2}, \\
& =\frac{4 \pi B_{0}^{2}}{\beta^{2}}(1-\cos \beta t), 4 \pi B_{0}^{2}<c^{2},
\end{aligned}
$$

where $\beta=\sqrt{8 \pi G \rho\left|1-2 \pi B_{0}^{2} / c^{2}\right| / 3}$. Since for the solution involving cosh, the radial function $R(t)$ is negative, we discard this solution as unphysical. For the remaining part of the solution we write the magnetic energy density $4 \pi B_{0}^{2}$, as the mean kinetic energy of expansion. Therefore, by virial theorem, we write $4 \pi B_{0}^{2}=2 V^{2}$, where $V^{2}$ is the average energy of expansion (per unit mass) per $M p c$. This gives

$$
R(t)=R_{0}(1-\cos \beta t), V^{2}<c^{2},
$$

where $\beta=\sqrt{8 \pi G \rho\left|1-V^{2} / c^{2}\right| / 3}$ and $R_{0}=V^{2} / \beta^{2}$.

The acceleration equation (10) also has a solution when $4 \pi B_{0}^{2}=c^{2}$. In this case $\beta=0$ and we have using $R(0)=0$,

$$
R(t)=2 \pi B_{0}^{2} t^{2} .
$$


Putting $B_{0}^{2}=c^{2} / 2 \pi$, by virial theorem, we obtain

$$
R(t)=c^{2} t^{2}, \text { when } \beta=0 .
$$

In this case the universe accelerates as $d^{2} R / d t^{2}=2 c^{2}$. This however implies that, for a non-zero matter density, the average speed of expansion $V$ must be equal to the speed of light, which is possible only in the inflation phase of expansion.

\section{Results}

Equation (13) implies a cyclic, oscillating universe. To derive the time and radius for maximum expansion, we see that the parameter $\beta$ depends on the matter density $\rho$ of the universe. Observations show that the matter density at present is approximately $\rho_{0}=\rho\left(t_{0}\right) \sim 10^{-26} \mathrm{~kg} / \mathrm{m}^{3}$. Also, the observed order of magnitude (up to the $M p c$ ) for the magnetic field is of $\mu G$, we have $V^{2}=4 \pi B_{0}^{2} \sim 10^{-11} \mathrm{~J} / \mathrm{m}^{3}$ valid at least up to a $M p c$, we can therefore neglect $V^{2} / c^{2}$ term in $\beta$. This gives for the expansion parameter $\beta=\sqrt{8 \pi G \rho / 3} \sim 21 \times$ $10^{-19} / \mathrm{s}$, which leads to the time for maximum expansion $t_{\max } \sim \pi / \beta \sim 10^{19} \mathrm{~s}$.

Also, equation (13) shows that there is a maximum expansion at $2 R_{0}=$ $2 V^{2} / \beta^{2}=4 \pi B_{0}^{2} / \beta^{2}$. Since $V^{2}$ is the energy density per unit mass, per unit distance of $M p c$, this gives, $V^{2} / \beta^{2} \sim 10^{5} M p c$. Therefore the maximum radius of expansion for the universe is approximately $200 G p c$. Figure 1 shows the scale function for the expansion phase of the universe, corresponding to the solution (13).

In equation (13) $\beta$ is the expansion rate, related to the Hubble's parameter $H_{0}=H\left(t_{0}\right)$ as follows. For the present matter density, parameter $H_{0}$ is related to $\beta$ by scaling as $\beta(M p c / k m) \approx H\left(t_{0}\right)$. Putting the value for $\beta$ (neglecting $V^{2} / c^{2}$ ), and using this scaling we obtain the Hubble parameter at present as

$$
H\left(t_{0}\right) \sim\left(21 \times 10^{-19} / \mathrm{s}\right)\left(3 \times 10^{22} / 10^{3}\right)=63(\mathrm{~km} / \mathrm{s}) \mathrm{Mpc}^{-1} .
$$

This value of the Hubbles's parameter however excludes the contribution of magneto-vorticity term $4 \pi B_{0}^{2}$ in the acceleration equation (10). For a better estimate we therefore use energy equation (3) directly. Putting back $c^{2}$ factor, we see that for a flat universe $H=H_{0}+H_{B}$, where $H_{0}=\sqrt{8 \pi G \rho_{0} / 3}=H\left(t_{0}\right)$ and $H_{B}=\sqrt{B_{0}^{2} c^{2} / 4 \pi R^{2}}$. On $M p c$ scale, $R=1 M p c$ and for average observed magnetic field $B_{0} \approx 10^{-7} G$. Also, interpreting $H$ as the total expansion velocity per $M p c$ with $H_{0}$ and $H_{B}$ as velocity components per $M p c$, we have measuring both $H_{0}$ and $H_{B}$ in $\mathrm{km} / \mathrm{s}$ per $M p c, H_{B}=8.8(\mathrm{~km} / \mathrm{s}) M p c^{-1}$. This gives for the present rate of expansion $H \approx 72(\mathrm{~km} / \mathrm{s}) \mathrm{Mpc}^{-1}$, which lies in the observed range of values for the Hubble's parameter (see e.g., Ref. [3-5]). Also, a relevant parameter to the expansion is the acceleration magnitude. We see from equation (13) that it is approximately given by

$$
a_{0}=\left|\frac{d^{2} R}{d t^{2}}\right|=\beta^{2} R_{0}=H^{2}\left(\frac{V^{2}}{H}\right)=3.9 \times 10^{5} \mathrm{~km}^{2} / \mathrm{s}^{2} M p c^{-1} .
$$


In Figure 2 we give the expansion rate $R(t)$ up to the $50 \mathrm{Mpc}$ of the observed universe, where the present time is $t=1$ on the horizontal scale. We notice that the accelerated expansion becomes significant after time scale of about $0.2 \times 10^{17} \mathrm{~s}$. This value corresponds to the expansion scale of $M p c$ for Type Ia supernovae $[28,29]$.

We now deduce the (virial) energy corresponding to this expansion (acceleration). Since the kinetic energy of expansion $K$ in a flat universe must be equal to the total potential energy $U$, we have by the energy balance for local matter distribution

$$
K=U_{B}+U_{G},
$$

which gives on taking average over cluster distributions in the universe,

$$
\langle K\rangle=\left\langle U_{B}\right\rangle+\left\langle U_{G}\right\rangle .
$$

By virial theorem, for gravitationally bound systems, $2\langle K\rangle+\left\langle U_{G}\right\rangle=0$, which implies that $\left\langle U_{G}\right\rangle=-2\langle K\rangle$. Therefore,

$$
\left\langle U_{B}\right\rangle=3\langle K\rangle .
$$

Since the dynamical mass is contained in the kinetic energy term, this implies that the magnetic field contributes 3 times more mass in the dynamics, hence expansion, of the universe than the gravitational mass. We see that this factor comes essentially from virial theorem, which confirms that the missing dynamical mass must be due to collective (average) effect of individual (galactic) masses.

Notice that the extent of extragalactic magnetic fields, for a cluster of galaxies, can be determined by the condition, $\cos (\varphi-\omega t)=0$. Given a time scale of 6 billion years for cosmic expansion, and an average angular momentum $J=m v r=10^{64} \mathrm{~m}^{2} \mathrm{~kg} / \mathrm{s}$ per galaxy in a cluster (consisting of a thousand galaxies), we see that for $\varphi=0$, (that is, along a given direction in space), we have

$J t / r^{3}=\pi / 2$. This implies that $r \sim 10^{27} \mathrm{~m} \sim 100 \mathrm{Gpc}$. This again is compatible with the spatial extent of maximum expansion derived above.

\section{Behavior near singularity}

In the contraction phase, we see that, with sufficient accumulation of mass density, Schwarzschild singularity $R_{s}$ begins to form. As $R \rightarrow R_{s}$, we see, however, that the energy density due to the magnetic field becomes increasingly small, since the logarithmic term in equation (2) becomes negative. Maxwell equations however imply that under spacetime reversal, the magnetic field should reverse sign as well. It therefore follows that magnetic field, hence the magnetic energy density, must be zero at $R=R_{s}$. However, such reversion implies that the gravitational attraction must become extremely large as $R$ becomes smaller. Contraction, therefore, continues up to the radius $R=R_{s}$ with an accelerated rate. Once cross-over at the Schwarzschild surface occurs, spacetime inversion 
must then cause both magnetic and gravitational fields to invert signs as well. For the interior spacetime we therefore have the magnetic energy density

$$
\epsilon_{B}=-4 \pi B_{0}^{2}\left[R+R_{s} \ln \left(R_{s}-R\right)\right], \quad R<R_{s},
$$

which now acts as an attractive source of energy, where as the gravitational field energy $\epsilon_{G}=-G M / R$ is now repulsive for $R<R_{s}$. On further contraction, as $R \rightarrow 0$, gravitational repulsive energy exceeds the magnetic attractive energy, and expansion starts in the region $R<R_{s}$. For this expansion we have the solution

$$
R(t)=R_{s}-R_{0}(\cosh \beta t-1), V^{2}<c^{2} .
$$

Quantum gravity effects, however, imply that the radius $R_{s}$ over which this sudden expansion occurs, due to very small length scale, lies below the Planck time $t_{p}$. Since $\epsilon_{B}\left(R_{s}\right)=0$, this expansion continues beyond $R=R_{s}$ (Figure 3 ), due to the gravitational force.

As the solutions posses symmetry, the magnetic energy density in the neighborhood of cross-over radius $R=R_{s}$ will have a very high degree of isotropy. Maximum expansion then corresponds to a very high degree of entropy around cross-over $R=R_{s}$ well into the region $R>R_{s}$. Initial radiation distribution therefore must be isotropic and of the Planckian profile due to the thermodynamic equilibrium between matter and radiation. After the initial gravitational expansion into the region $R>R_{s}$, as $R$ increases, magneto-vorticity effects in spacetime begin to dominate after a time period of Planck time scale. However, gravity is now attractive, therefore a shock like condition must result causing matter accumulation, and vorticity generation.

\section{Conclusions}

To recapitulate we remark that, within the above model of the accelerated expansion of the universe, local spacetime vorticity and magnetic field energy generation within galaxies and galactic clusters act as the feedback mechanism for expansion. Thus contrary to some recent suggestions that accelerated expansion must imply a violation of the law of conservation of energy, we see that energy conservation remains strictly valid not only locally but globally as well. The continued universal acceleration depends on the energy generation within galaxies, which in turn is determined by accretion rate in galactic nuclei. Conversion of matter-energy density into the magnetic field energy under such conditions can only take a finite amount of time, hence the magnetic field driven acceleration cannot continue indefinitely for a finite total mass. Since the acceleration $a_{B} \sim B_{0}^{2}$, where $B_{0}^{2}$ is the magnetic energy density per unit volume, we see that with the decreasing feedback magnetic field, universal acceleration after reaching an maximum will gradually decrease. With the decrease of magnetic energy generation via accretion, a gradual deacceleration under gravitational attraction is likely to cause cosmic contraction, as shown above. We therefore have an oscillatory universe, where magneto-vorticity coupling rather than global spacetime curvature causes the expansion and contraction phases. 
Finally, a very high degree of entropy must have existed at the early stage of the universe, as inferred from the Planckian shape of the CMB radiation. This raises the paradox for other cosmological models, since entropy should decrease closer to the initial singularity (big bang). The above model implies that this must be so due to the expansion started before the cross-over $R=R_{s}$. Subsequently, as this expansion (inflation) stops, and matter formation starts, expansion under spacetime vorticity must now cause matter entropy to gradually increase with time. As deduced above this explains the high isotropy and the Planckian profile of the CMB spectrum, carrying the imprint of this initial inflation over $R>R_{s}$.

\section{References}

[1] Riess, A. G., Filippenko, A. V., Challis, P., Clocchiatti, A., Diercks, A., et al. 1998, AJ 116, 1009

[2] Perlmutter, S., Aldering, G., Goldhaber, G., Knop, R. A., Nugent, P., et al. 1999, ApJ, 517, 565

[3] Jackson, N. 2015, Living Reviews in Relativity, 18, 2

[4] LIGO Scientific Collaboration, et al. 2017, Nature 551(7678), 85

[5] Di Valentino, E, Melchiorri, A. 2018, Physical Review D 97(4), 041301

[6] Halverson, N. W., Leitch, E. M., Pryke, C., Kovac, J., Carlstrom, J. E., et al. 2002 ApJ, 568, 38

[7] Bennett, C. L., Halpern, M., Hinshaw, G., Jarosik, N., Kogut, A., et al. 2003 ApJS, 148, 1

[8] Seo, H. J., \& Eisenstein, D. J. (2003). ApJ, 598, 720

[9] Dawson, K. S., Schlegel, D. J., Ahn, C. P., Anderson, S. F., Aubourg, É., 2012 AJ, 145, 10

[10] Ade, P. A., Aghanim, N., Arnaud, M., Ashdown, M., Aumont, J., et al. 2016, A\&A, 594, A13

[11] Rubin, D., \& Hayden, B. 2016, ApJL, 833, L30

[12] Larson, D., Weiland, J. L., Hinshaw, G., \& Bennett, C. L. 2015, ApJ, 801, 9

[13] Spergel, D. N., Bean, R., Doré, O., Nolta, M. R., Bennett, C. L., et al. 2007, ApJS, 170, 377

[14] Ade, P. A. R., Aghanim, N., Armitage-Caplan, C., Arnaud, M., Ashdown, M., et al. 2014 A\&A, 571, A16.11 
[15] de Bernardis, P., Ade, P. A., Bock, J. J., Bond, J. R., Borrill, J., et al. 2000, Nature, 404, 955

[16] Rubin, D., Aldering, G., Barbary, K., Boone, K., Chappell, G., 2015 ApJ, 813,137

[17] Beck, R., \& Wielebinski, R. 2013, in Planets, Stars and Stellar Systems (Netherlands: Springer), pp. 641-723

[18] Kronberg, P. P., Kothes, R., Salter, C. J., \& Perillat, P. 2007, ApJ, 659, 267

[19] Beck, R. 2011, AIP Con.Proc. 1381, 117

[20] Xu, Y., Kronberg, P. P., Habib, S., \& Dufton, Q. W. 2006, ApJ, 637, 19

[21] Widrow, L. M. 2002, Rev. Mod. Phys., 74, 775

[22] Taylor, G. B., Barton, E. J., \& Ge, J. 1994, AJ, 107, 1942

[23] Kim, K. T., Kronberg, P. P., Dewdney, P. E., \& Landecker, T. L. 1990, ApJ, 355, 29

[24] Kim, K. T., Tribble, P. C., \& Kronberg, P. P. 1991, ApJ, 379, 80

[25] Clarke, T. E., Kronberg, P. P., \& Böhringer, H. 2001, ApJL, 547, L111

[26] Kim, K. T., Kronberg, P. P., Giovannini, G., \& Venturi, T. 1989, Nature, 341,720

[27] Mirza, B. M. 2017, ApJ, 847, 73

[28] Riess, A.G., Li, W., Stetson, P.B., Filippenko, A.V., Jha, S., Kirshner, R.P., et al. 2005 ApJ, 627, 579

[29] Hoffmann, S.L., Macri, L.M., Riess, A.G., Yuan, W., Casertano, S., et al. 2016, ApJ, 830, 10

Figure Captions:

Figure 1: Accelerating expansion during the present phase up to the distance of $50 \mathrm{Mpc}$, where acceleration effects become significant for redshift observations of distant Type Ia supernovae.

Figure 2: Scale factor for expansion of the universe. Accelerating expansion is greatest during the first phase, lasting up to $0.4 \times 10^{19} \mathrm{~s}$, after which acceleration is almost zero for next $0.2 \times 10^{19} \mathrm{~s}$ and then decreases, reaching maximum expansion.

Figure 3: Expansion during the first Planck second $\left(=5.4 \times 10^{-44} s\right)$ of the universe, extending beyond the cross-over radius $R=R_{s}$. Energy density increase causes matter formation which subsequently slows the expansion under gravitational attraction. 
This figure "Fig_1.JPG" is available in "JPG" format from: http://arxiv.org/ps/1803.09628v4 
This figure "Fig_2.JPG" is available in "JPG" format from: http://arxiv.org/ps/1803.09628v4 
This figure "Fig_3.JPG" is available in "JPG" format from: http://arxiv.org/ps/1803.09628v4 